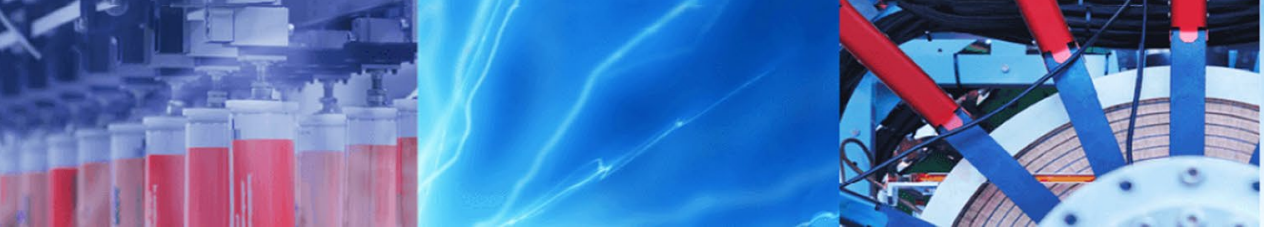

Research Article

\title{
A local Multivariate Polynomial Regression approach for ionospheric delay estimation of single-frequency NavIC receiver
}

\author{
Mehul V. Desai ${ }^{1}$ (D) . Shweta N. Shah ${ }^{1}$ (D)
}

Received: 23 April 2020 / Accepted: 23 July 2020 / Published online: 12 August 2020

(c) Springer Nature Switzerland AG 2020

\begin{abstract}
In this paper, we implemented the local Multivariate Polynomial Regression (MPR) approach to measure the ionospheric delay more accurately for single-frequency Navigation with Indian Constellation (NavlC) system within a local area $(<10 \mathrm{~km})$. The idea is, if the reference station can transmit the ionospheric correction to the rover station, then the rover receiver can also estimate the ionospheric delay accurately just as a dual-frequency reference station without having dual-frequency hardware facility. In addition to ionospheric correction, to enhance the positioning accuracy of the single-frequency rover NavIC receiver, the tropospheric and clock biases are also corrected. Including MPR, other single-frequency global eight coefficient Klobuchar, regional grid-based GIVD, and local proposed TSE approach are also applied and correlates with the reference dual-frequency method. Based on the geomagnetic index and data availability of the two NavlC Receiver, quiet $\left(0<K_{p}<1\right)$, disturbed/stormy $\left(K_{p}>5\right)$ days are selected for the investigation. The results of NavIC ionospheric delay and 3D positioning error (East, North, and Up) show that the proposed MPR method worked well compared to the other applied single-frequency models.
\end{abstract}

Keywords Navigation with Indian constellation (NavIC) - Indian regional navigation satellite system (IRNSS) . lonospheric delay · Geomagnetic storm · Positioning accuracy

\section{Introduction}

The Indian Regional Navigation Satellite System (IRNSS), with an operational name NavIC ("sailor" or "navigator" in Sanskrit), has been established by Indian Space Research Organization (ISRO) to impart the service to military and civilian users in any antagonistic situations toward the Indian subcontinent and extended up to $1500 \mathrm{~km} \mathrm{[12].}$ The system of seven satellites NavIC utilizing L5-band (1164.45-1188.45 MHz) and S-band (2483.5-2500 MHz) signals to impart Special Positioning Service (SPS) and Precision Service (PS) toward the Indian subcontinent. There are currently seven NavIC satellites, 1B-1G, and 11 providing navigation services [13].
Efficient, robust, and precise NavIC receivers are crucial for future Internet of Things (IoT) positioning and other safety-critical applications. However, the satellite signals received by the NaviC receiver are affected by many artificial error sources such as Jammer and existing systems with the same frequency band $[14,16]$. Similarly, it is also affected by the natural error sources like satellite atomic clocks, ephemeris (satellite orbital information), atmospheric delays, receiver clock drift, and noise [1-7]. The ionosphere is a spherically graded layer that contains large electron density and is the main source of errors for ranging and localization using NavIC frequencies. The extent of deviation of a NavlC signal propagating through the ionosphere depends on its large irregularities and horizontal gradients, the height of the geographic location,

Mehul V. Desai, mvd.svnit@gmail.com | ${ }^{1}$ Electronics Engineering Department, Sardar Vallabhbhai National Institute of Technology, Surat, India. 
the daily variation, the seasonal variation, and the solar activity $[3,5,6]$.

To boost GPS positioning accuracy and expand coverage, India has developed a Satellite-Based Augmentation System (SBAS), commonly referred to as GPS-Aided Geo Augmented Navigation (GAGAN). Many authors have reported that the performance of the GAGAN system is more influenced by the lower latitude equatorial Indian ionosphere because of its large gradients and strong irregularities [17-25]. They proposed various regional models because the global traditional Klobuchar model could not estimate the ionospheric delay of the Indian subcontinent adequately.

According to Fujita et al., TEC can be accurately estimated by dual-frequency methods during storms, seasonal activities, and daily activities. Still, it is challenging to do this with single-frequency global and regional models [8, 9]. Similarly, to accurately estimate TEC at low latitudes in India, P. Naveenkumar et al. (2014) [15] a local Taylor Series Expansion (TSE) model was implemented on the GAGAN system. They found that, in contrast to the global Klobuchar model, the local model performed better on quiet days than on the disturbed days of all 17 observed stations [15]. Their results indicate that the model is outperforming for quiet days than the disturbed days. This has enlightened the idea that "at the lower latitude Indian region also the large temporal and spatial ionospheric gradients are present. Therefore, to enhance the positioning accuracy of the Navigation system, the local model is essential."

Therefore, we [5] proposed, the TSE approach to estimate the precise ionospheric delay of a single-frequency rover NaviC receiver within a local region $(<10 \mathrm{~km})$. In this case, we assume that the dual-frequency reference NavlC receiver broadcasts the ionospheric coefficient, which is estimated from the measured Vertical Total Electron Content (VTEC) value for the NavIC satellites, and based on the broadcast coefficients, the single-frequency rover NavIC receiver can estimate ionospheric delay accurately. We examined the local TSE approach performance under the influence of the intense geomagnetic storm $\left(D_{s t}=-124\right.$, $K_{p}=8, A_{p}=106$ ) on September 8, 2017, on the equator and Equatorial lonization Anomalies (EIA) in India. We infer that due to the six asymmetry coefficients, the performance of the TSE method will deviate within a certain period of time. To overcome this deviation, in this paper, we proposed 'Multivariate Polynomial Regression (MPR)' with six symmetrical coefficients to estimate the ionospheric delay of rover NavIC receivers. The NavIC 3D positioning errors of the proposed MPR approach are compared with the local TSE approach [5], regional grid-based Grid lonospheric Vertical Delay (GIVD) [7], global Klobuchar model [2], and reference dual-frequency method.

In addition, considering the effects of changing the position of the rover within the $10 \mathrm{~km}$ zone, the performance of the proposed TSE and MPR approaches are also examined for various quiet and disturbing days data. From the analysis of results, we concluded that the performance of the singlefrequency proposed TSE and MPR approaches differs from the performance of the reference dual-frequency model by only $\sim 5 \%$, which is better than regional grid-based GIVD model and the global Klobuchar model. MPR eliminates the spikes that appear in the TSE approach. Therefore, the proposed MPR approach not only accurately measures the ionospheric delay of the local region, but also reduces the hardware cost for the extra frequency.

\section{Multivariate polynomial regression model}

Polynomial regression model uses multiple independent variable $X$ and one dependent variable $y$ then it is called multiple polynomial regression model. Similarly, when it uses various dependent as well as an independent variable called MPR model. The second-order multiple polynomial regression functional equation can be represented as (Sinha et al. 2013b):

$E(y)=\beta_{0}+\beta_{1} X_{1}+\beta_{2} X_{2}+\beta_{11} X_{1}^{2}+\beta_{22} X_{2}^{2}+\beta_{12} X_{1} X_{2}+\varepsilon$

Here,

$\beta_{1}$ and $\beta_{2}$ parameters show the linear effect,

$\beta_{11}$ and $\beta_{22}$ show the quadratic effect,

$\beta_{12}$ shows the interaction effect,

$\varepsilon$ shows the noise.

Similarly, the model having multiple dependent variable $Q=\left(y_{1}, y_{2} \ldots y_{n}\right)$ can be represented in matrix form as: $Q=P B+\varepsilon$

where $P$ is the matrix of multiple independent variable $X$. The solution can be obtained by applying the least square estimation approach as,

$\hat{B}=\left(P^{T} P\right)^{-1} P^{T} Q$

The mathematical steps and case studies using the proposed MPR method to estimate the ionospheric delay of a single-frequency rover receiver are similar to those explained by [5], the only difference in generated coefficients.

\section{Test setup and data collection}

The NavIC satellite data were collected using the Accord NaviC receiver, which is provided by Space Application Center (SAC), ISRO Ahmedabad, India. The setup consists of an antenna that receives the NavlC signal at both 
frequency bands (L5-band and S-band) and GPS L1-band signals. Jagiwala et al. reported, as well as we observed, that NavIC S-band is sensitive to intentional and unintentional error sources [14]. Hence, here NavIC L5-band is explored for the ionospheric delay and positioning error analysis.

The test set up and flow diagram of the proposed approach are explained in [5]. The proposed local MPR approach coefficients are generated based on the VTEC measured by the reference dual-frequency NavIC receiver. The single-frequency rover receivers attempt to estimate the VTEC followed by ionospheric delay using the proposed local approach coefficients produced by the reference dual-frequency receiver. This analysis takes into account two Cases for proposed approach validation, which are explained below.

\subsection{Case-I}

Here, the proposed approach is analyzed by the single static NavlC receiver. This means that the proposed approach coefficients generation and the VTEC estimation (using the proposed approach coefficients) are carried out for static single NavIC receiver. In Case (I), the proposed local TSE and MPR approaches results are examined during a quiet day 04/09/16 $\left(K_{p}=4+, A_{p}=27\right)$. The result is also observed on intense (Dst $=-124, K_{p}=8$,
$\left.A_{p}=106\right)$ geomagnetic storm (September 8, 2017) using NavIC receiver data collected over a week (September $3-9,2017)$ at the geographical locations mentioned in [5]. Here, the ionospheric delay for single-frequency NavIC is computed using global Klobuchar, regional GIVD model, and proposed local TSE and MPR approaches. Finally, the performance of the single-frequency model is validated by calculating the residual error.

\subsection{Case-2}

In Case (II), two NaviC stations are considered, one of them is the reference station equipped with dual-frequency hardware, and another one works as a rover station with single-frequency hardware.

The idea is that if the bias-free dual-frequency reference station can transmit the ionospheric correction to the single-frequency rover station, then the rover receiver can also estimate the ionospheric delay accurately just as the dual-frequency reference station without having dualfrequency facility. It is assumed that the reference station can transmit the coefficients similar to the Differential Global Positioning System (DGPS).

The $10 \mathrm{~km}$ of the local region is considered and based on proposed TSE, and MPR approaches are analyzed by considering two separate NavlC receivers. As shown in Fig. 1, the reference station (marked with pink color) is

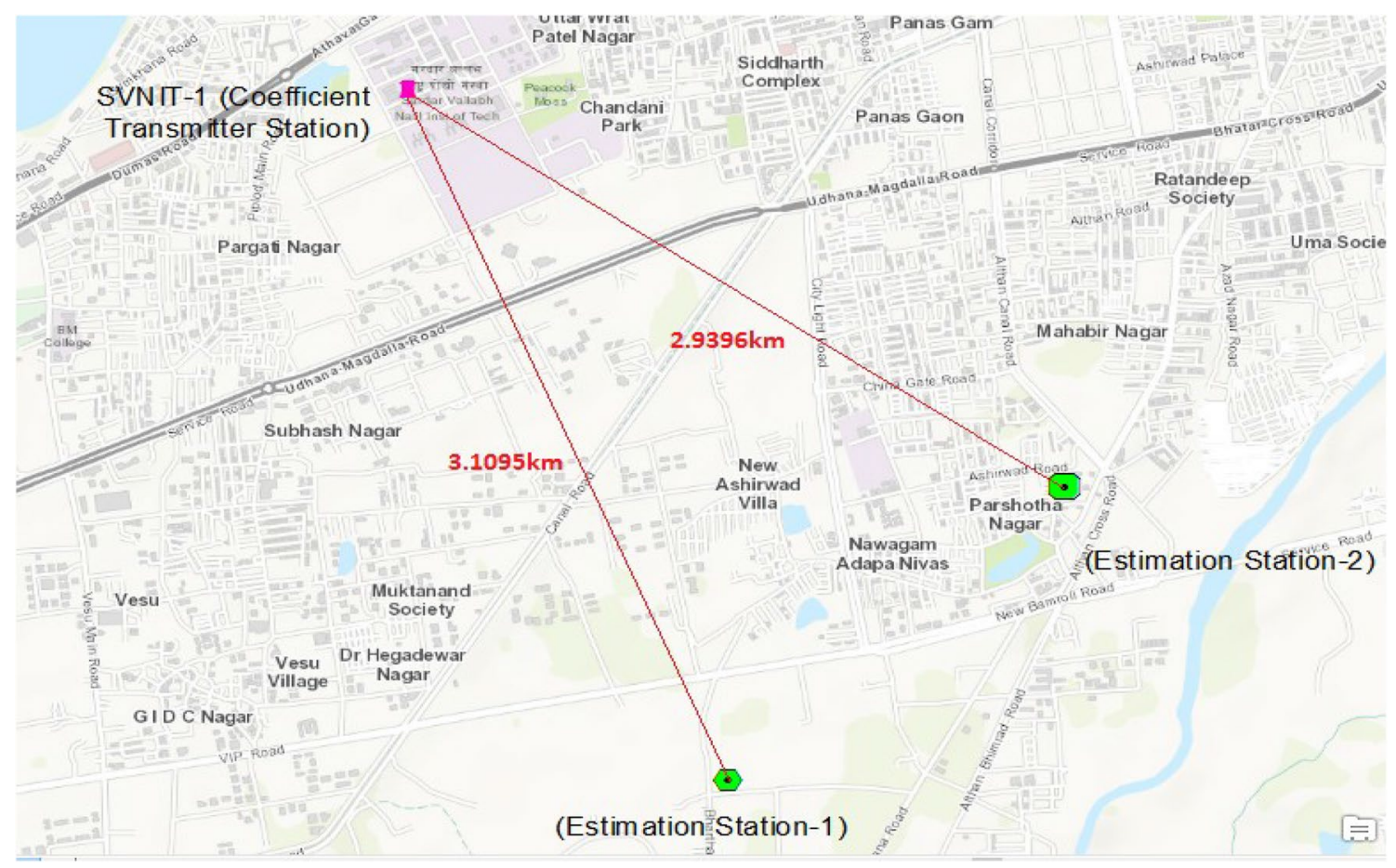

Fig.1 Station Physical Location in ArcMap10.3 
selected as the coefficients generating station, which is located at the Communication Research Laboratory (CRL), Electronics Engineering Department, Sardar Vallabhbhai National Institute of Technology (SVNIT), Surat $\left(21^{\circ}\right.$ $9^{\prime} 50.0926^{\prime \prime} \mathrm{N}, 72^{\circ} 47^{\prime} 1.2511^{\prime \prime} \mathrm{E}$ ) and the rover stations marked by green color are used for the analysis. The rover station uses the coefficients to estimate the TEC and thereby the ionospheric delay.

As depicted in Fig. 1, the direct distances between reference SVNIT station and rover station- 1 and rover station-2 (refer Table 1 are approximately $3.1 \mathrm{~km}$ and $2.9 \mathrm{~km}$, respectively (ArcMap 10.3). Further, quiet and disturbed days data are selected for the analysis, which is usually available for all receivers. To verify the single-frequency Klobuchar, results of the regional GIVD and proposed local TSE and MPR approaches are correlated with the reference model in terms of ionospheric delay as well as positioning accuracy. The simulation of the proposed approach is explained in the next section.

\section{Numerical results and discussion}

This section validates the theoretical analysis of the proposed approaches as well as the procedure explained in the above subsection. The MATLAB R.14 tool is used to estimate the ionospheric delay and $3 \mathrm{D}$ positioning error for the NavIC L5-band.

\subsection{Case-I: single receiver}

The ionospheric delay of six NavIC (1B to 1G) satellites is estimated using proposed local TSE, and MPR approaches at the reference SVNIT, Surat station on a quiet day $\left(04 / 09 / 16, K_{p}=4+, A_{p}=27\right)$. We have not removed ephemeris errors and multipath deviations from NavIC L5-band and S-band pseudoranges. Therefore, the ionospheric delay calculated using the dual-frequency method may not be correct, while the ionospheric delay estimated using the grid model has no multipath effect. Therefore, to verify the performance of the proposed TSE and MPR approaches, two subcases (a) and (b) are considered. In case-a, the proposed approach coefficients are generated using the dual-frequency method and regarded as a reference model.

Table 1 The Position of NavIC Reference and Rover Receivers

\begin{tabular}{lll}
\hline Station Name & Latitude & Longitude \\
\hline Reference Receiver & $21^{\circ} 9^{\prime} 50.0926^{\prime \prime} \mathrm{N}$ & $72^{\circ} 47^{\prime} 1.2511^{\prime \prime} \mathrm{E}$ \\
Rover Station-1 & $21^{\circ} 8^{\prime} 23.5993^{\prime \prime} \mathrm{N}$ & $72^{\circ} 47^{\prime} 39.6024^{\prime \prime} \mathrm{E}$ \\
Rover Station-2 & $21^{\circ} 9^{\prime} 20.7590^{\prime \prime} \mathrm{N}$ & $72^{\circ} 49^{\prime} 20.1275^{\prime \prime} \mathrm{E}$ \\
\hline
\end{tabular}

In Case b, a single-frequency GIVD model is used to generate the proposed approach coefficients, and it is treated as a reference model. lonospheric delay performance comparison of the proposed local TSE and MPR approaches, dual-frequency, global Klobuchar, and regional GIVD model for the NavIC 1D and 1G satellites at reference SVNIT surat station on a quiet day (04/09/16, $\mathrm{K}_{\mathrm{p}}=4+, \mathrm{A}_{\mathrm{p}}=27$ ) is compared in Figs. 2 and 3, respectively. Here, legends TSE-Dual and MPR-Dual indicate that the proposed approach coefficients were estimated using the dual-frequency method. TSE-GIVD and MPR-GIVD suggest

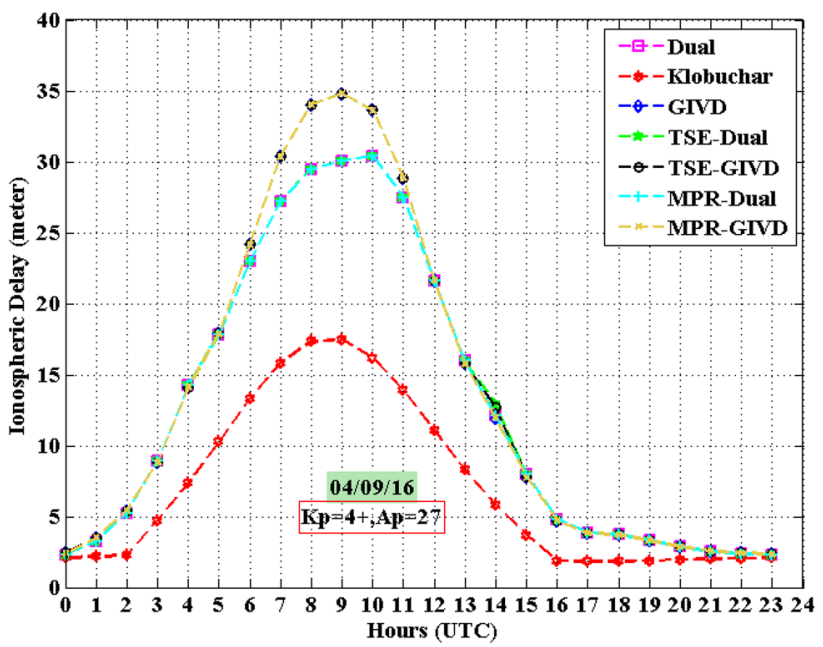

Fig. 2 lonospheric Delay Comparisons of NavIC 1D L5-Band satellite estimated using dual-frequency method and various single-frequency models/approaches on 04/09/16 $\left(K_{p}=4+, A_{P}=27\right)$ at Reference SVNIT Surat Station

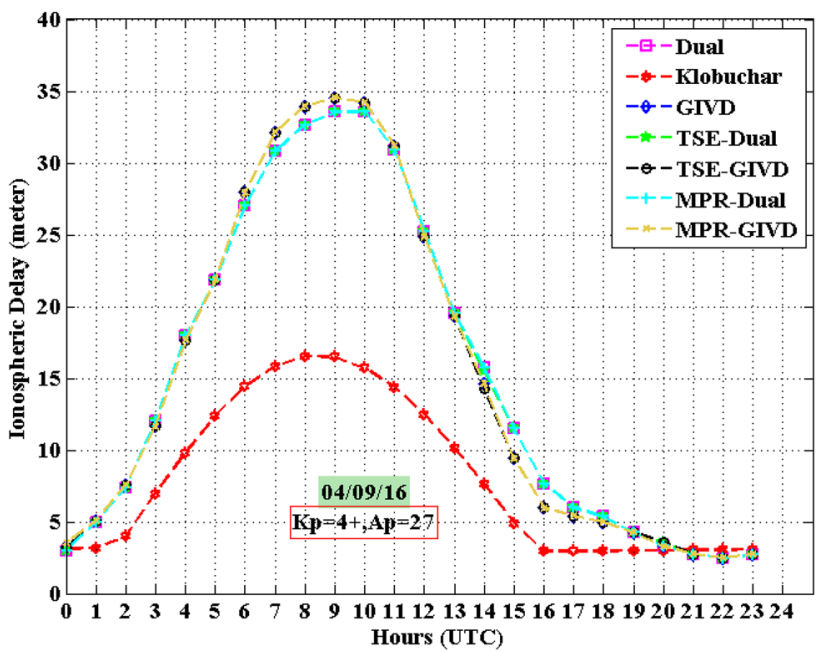

Fig. 3 lonospheric delay comparisons of NaviC 1G L5-Band satellite estimated using dual-frequency method and various single-frequency Models/Approaches on 04/09/16 $\left(K_{p}=4+, A_{p}=27\right)$ at Reference SVNIT Surat Station 
that the coefficients were generated using the single-frequency GIVD model at the reference receiver.

As shown in Figs. 2 and 3, we can observe that in both cases, the performance of the proposed local TSE and MPR approaches are similar to the reference model. The results of proposed approaches are also verified for other geographical locations mentioned in [5] under the effects of an intense geomagnetic storm (maximum $D_{s t}=-124$, $K_{p}=8, A_{p}=106$ ) beginning from September 8, 2017. The proposed local TSE and MPR approaches are validated at the observed stations considering one-week data from September 3-9, 2017.

In Fig. 4, the ionospheric delay of NavIC 1D satellite estimated using dual-frequency, GIVD model, and local proposed approaches were compared for two cases (a and b) at SVNIT Surat on September 3-9, 2017. For both cases, we found that the proposed approaches estimate the ionospheric delay similar to the reference ionospheric delay model (either dual-frequency or single-frequency GIVD model).

The five days' (September 4-8, 2017) average ionospheric delay difference (ionospheric delay of reference model- ionospheric delay of the applied model) is computed for all observed stations. Figures 5 and 6 show the ionospheric delay difference calculated for reference dualfrequency method and reference single-frequency GIVD model, respectively. We found that the single epoch sigma of the difference between the dual-frequency method and the GIVD model is $1.39 \mathrm{~m}$ in both cases. It may be due to deviations from multipath, ephemeris errors, and noise. We also noted that using the reference GIVD model, the single-epoch sigma of the ionospheric delay difference of the proposed TSE and MPR methods is reduced compared to the reference dual-frequency method.

The five days' (September 4-8, 2017) average ionospheric delay of NaviC satellites were also calculated for the geographical location IIT Gandhinagar, IIT Bombay, CBIT Hyderabad, and IIST Trivandrum. In Case-a, we found that the single epoch sigma (ionospheric delay difference) of the proposed local TSE and MPR approaches at IIST Trivandrum, CBIT Hyderabad, IIT Bombay, and IIT Gandhinagar stations are only $0.12 \mathrm{~m}, 0.34 \mathrm{~m}, 0.42 \mathrm{~m}$, and $0.92 \mathrm{~m}$, respectively. Similarly, in the case- $b$, the single epoch sigma (ionospheric delay difference) of the local TSE and MPR of IIST Trivandrum, CBIT Hyderabad, IIT Bombay, and IIT Gandhinagar stations are close to $0.11 \mathrm{~m}, 0.07 \mathrm{~m}$, $0.14 \mathrm{~m}, 0.16 \mathrm{~m}$, respectively.

Therefore, we found that the ionospheric delay estimated by the proposed local TSE and MPR methods, GIVD, and dual-frequency models is almost the same on all observed quiet and disturbed days at all observation stations. However, due to the uncorrected multipath biases,

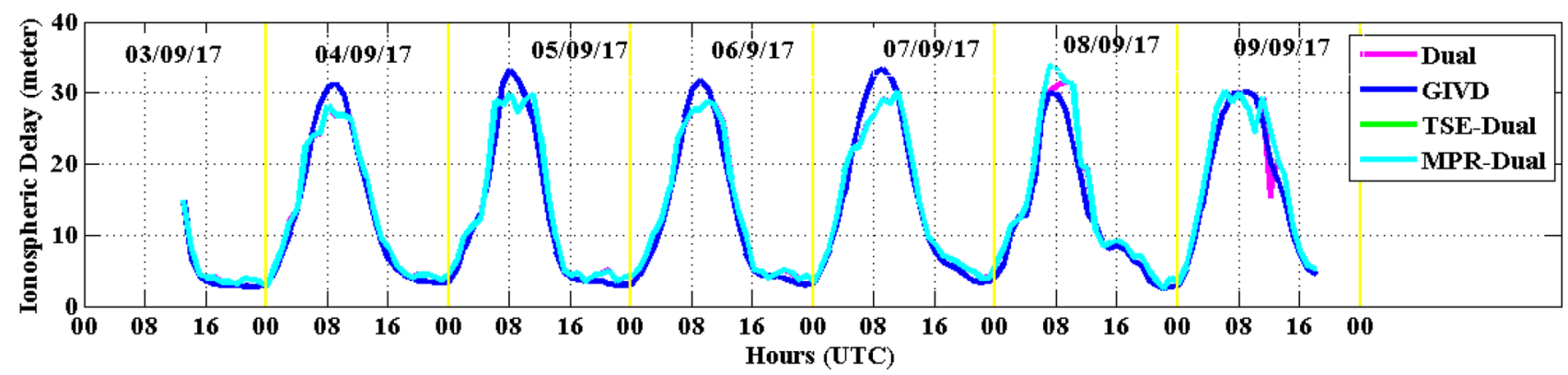

(a) Dual-frequency as a Reference

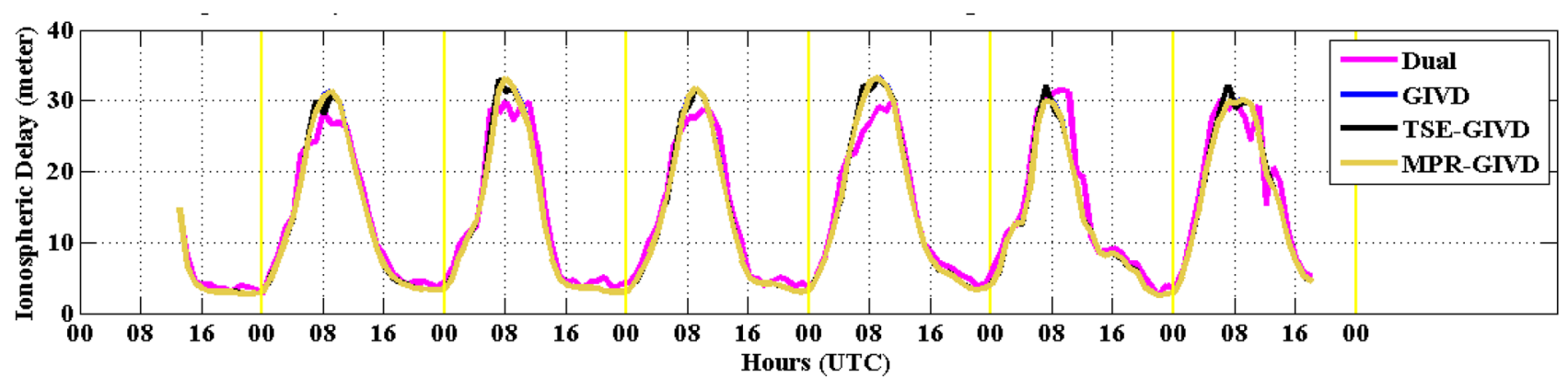

(b) GIVD Model as a Reference

Fig. 4 lonospheric Delay Comparisons of NavIC 1D L5-Band Satellite Estimated using dual-frequency method, single-frequency GIVD model and proposed TSE and MPR approaches by consider- ing reference as a a dual frequency $\mathbf{b}$ GIVD model on September 3-9, 2017 at reference SVNIT Surat Station 


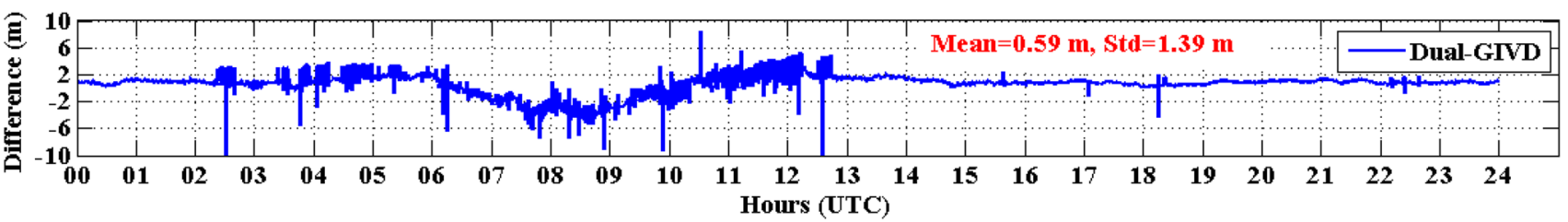

(a) Dual-frequency - GIVD Model

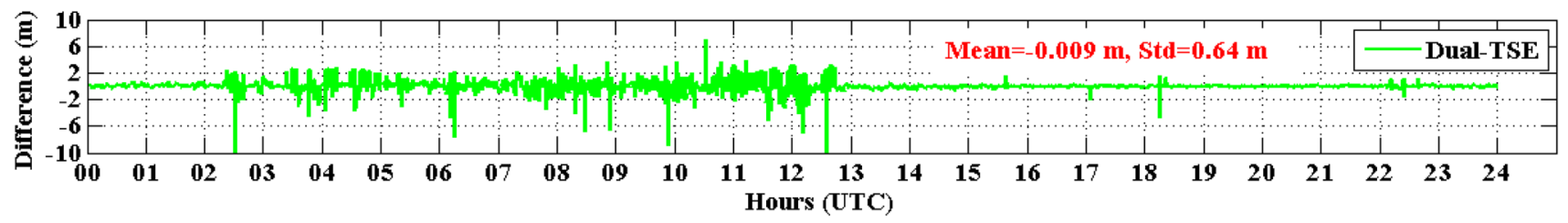

(b) Dual-frequency - TSE Approach

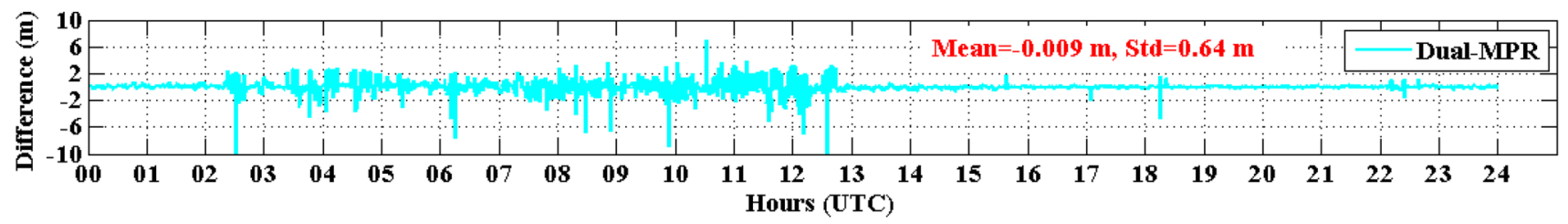

(c) Dual-frequency - MPR Approach

Fig. 5 lonospheric delay difference (Reference Model-Applied Model) for a GIVD Model b TSE Approach c dual-MPR approach of NavIC 1D L5-Band satellite considering dual-frequency method as a reference model on September 3-9, 2017 at reference SVNIT Surat Station

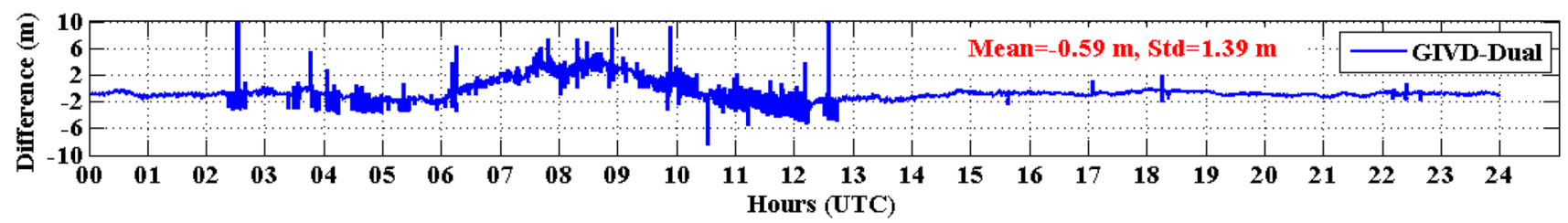

(a) GIVD Model- Dual-frequency

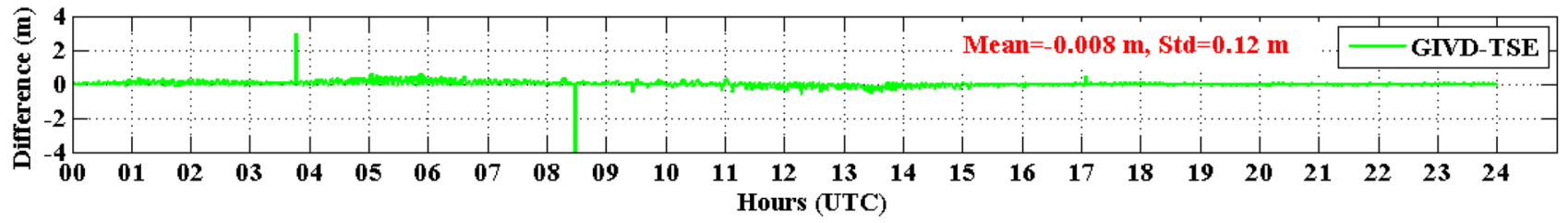

(b) GIVD Model- TSE Approach

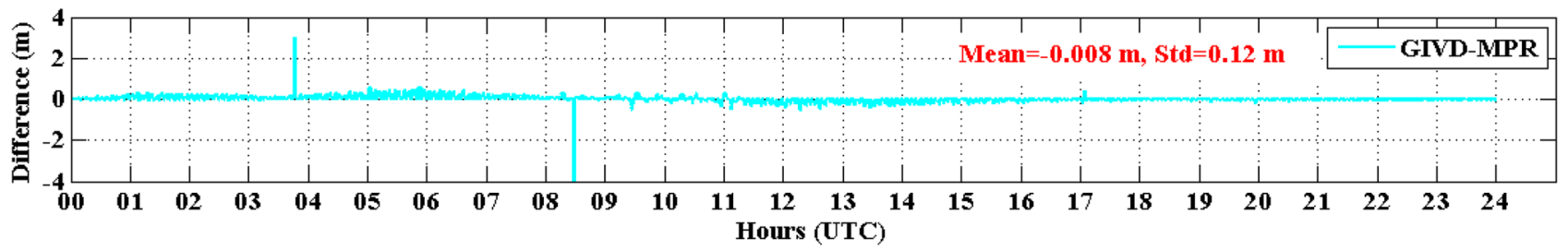

(c) GIVD Model- MPR Approach

Fig. 6 lonospheric delay difference (Reference Model-Applied Model) for a GIVD Model b TSE Approach c dual-MPR approach of NavIC 1D L5-Band satellite considering GIVD model as a reference model on September 3-9, 2017 at reference SVNIT Surat Station

for case-a, the residual error of the proposed local TSE and MPR approaches are higher than case-b. Hence, for Case-l, by observing the results of all observed geographic locations, we concluded that the proposed local TSE and MPR approaches could estimate ionospheric delay the 
same as the reference dual-frequency reference receiver even in the presence of the intense geomagnetic storm.

\subsection{Case-II: two receivers}

According to Case-Il, data from two NavIC receivers are required for the proposed approach performance validation, where both the NavIC receivers have to be with the same specifications and have to consider their hardware biases. We did not remove the ephemeris error and multipath from pseudorange. To reduce the multipath effects from the objects of surrounding environment, both receivers are located on top of the building. We choose two dualfrequency Accord NavlC receivers with their corrected biases like clock corrections and clock drift etc. available from NavIC L5/S band observation file (SATB L5/S.csv) and Raw navigation file (RNBB.csv).

First, we verified the performance of the proposed local TSE and MPR approaches at both the rover stations and estimates the ionospheric delay as well as the position of the rovers. The ionospheric delay of the NavIC satellites are calculated at rover station- 1 on the $04 / 09 / 16\left(K_{p}=4+\right.$, $\left.A_{P}=27\right)$ using the proposed local TSE, and MPR approaches for the cases $a$ and $b$ as discussed before. Here, the day for the analysis is selected based on the data availability of the two NavlC receivers within a $10 \mathrm{~km}$ area. The ionospheric delay of satellites computed using the local proposed approaches for the Cases $a$ and $b$ are compared with reference dual-frequency and GIVD model.

Similar to Case I, we observed that NavIC 1D and 1G satellites were more affected by the ionosphere than the other NavIC satellites due to its low elevation angle and their relative position in the sky. Therefore, the ionospheric

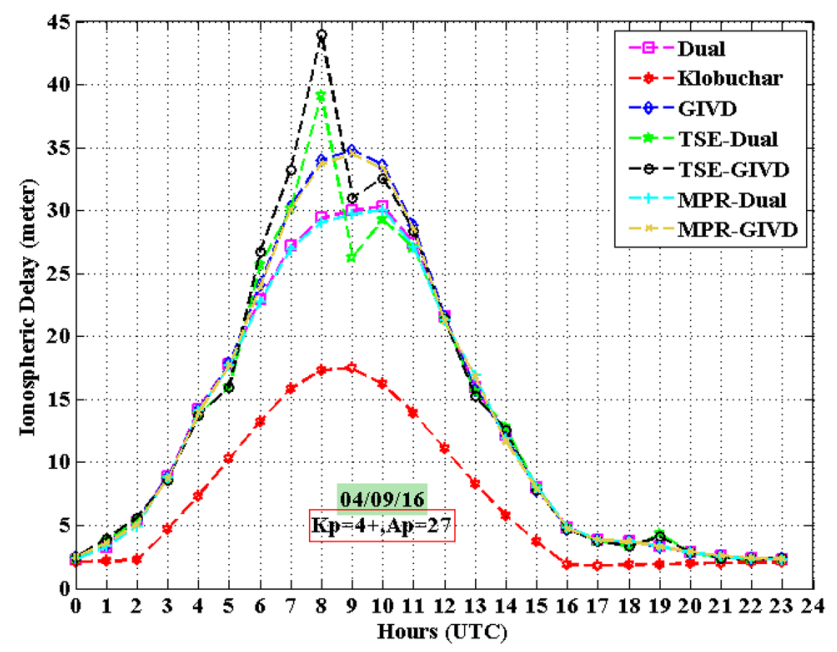

Fig. 7 lonospheric Delay Estimation Comparison for NavIC 1D Satellite at Rover Station 1 (21.130 N, 72.790 E) on 04/09/16 ( $K_{p}=4+$, $\left.A_{P}=27\right)$

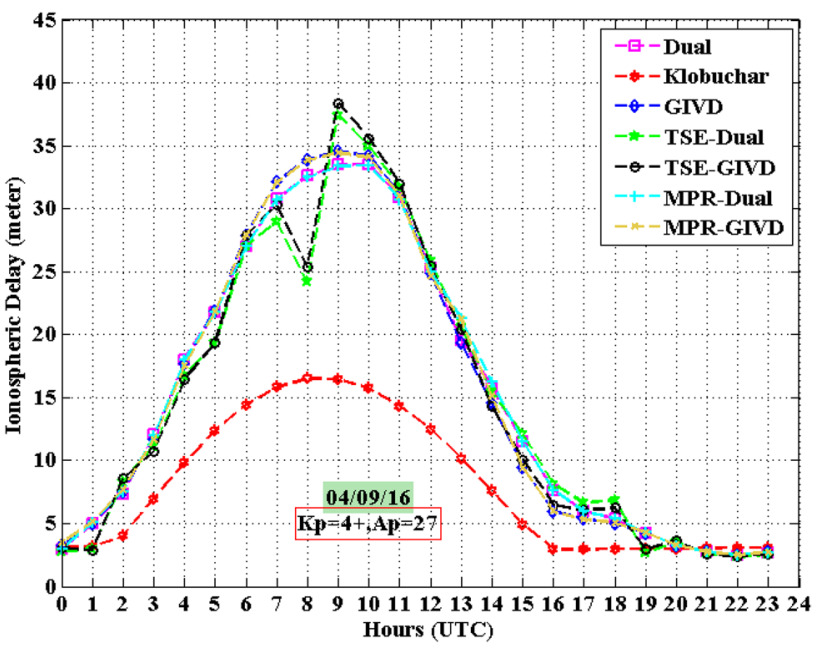

Fig. 8 lonospheric Delay Estimation Comparison for NaviC 1G Satellite at Rover Station-1(21.130 N, 72.790 E) on 04/09/16 ( $\mathrm{K}_{\mathrm{P}}=4+$, $A_{P}=27$ )

delays estimated using single-frequency models (global Klobuchar and regional GIVD), proposed local TSE and MPR approaches, and the dual-frequency method are compared for 1D and 1G satellites in Figs. 7 and 8, respectively. We observed that for both the cases ( $a$ and $b$ ), the proposed local MPR approach provides results similar to the reference model. On the contrary, the proposed local TSE method behavior is unstable between 8:00 and 10:00 (UTC) due to six-coefficient expansion is not symmetrical. In addition, the global Klobuchar model effectively evaluated only $50 \%$ of ionospheric delay, and the grid-based regional GIVD model ionospheric delay has differed to the dual-frequency model from 6:00 to 11:00 UTC (refer to Figs. 7 and 8).

A similar observation is made for the remaining NavlC satellites as well. To analyze the effect of the ionospheric delay on NavIC L5-band positioning error, the 3D position is calculated by the ILS estimation algorithm [11] after correcting the ionospheric effect, tropospheric effect and the satellite clock correction. The average rover NavIC receivers position is obtained by averaging the estimated position of the rover receiver, and it is considered as an actual position of the rover NaviC receivers.

The reference position of the rover receivers is listed in Table 1. Here, the troposphere delay for the NavIC L5-band signal is estimated by the Hopfield model [10]. The NavIC $3 \mathrm{D}$ positioning errors (on 04/09/16, TOWC $=0 \mathrm{~s}$ to $86,400 \mathrm{~s}$ ) in terms of the East, North, and Up coordinate system are estimated using (1) the global Klobuchar correction (2) the regional GIVD correction (3) the proposed local TSE approach correction (4) the proposed local MPR approach correction (5) the dual-frequency correction and (6) the augmented NavlC with GPS. 


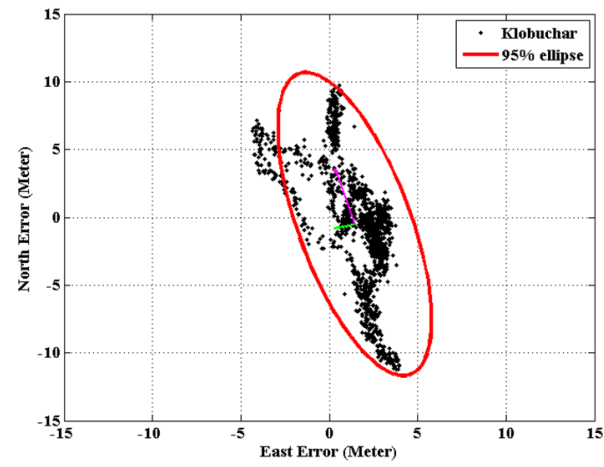

(a) Klobuchar Correction

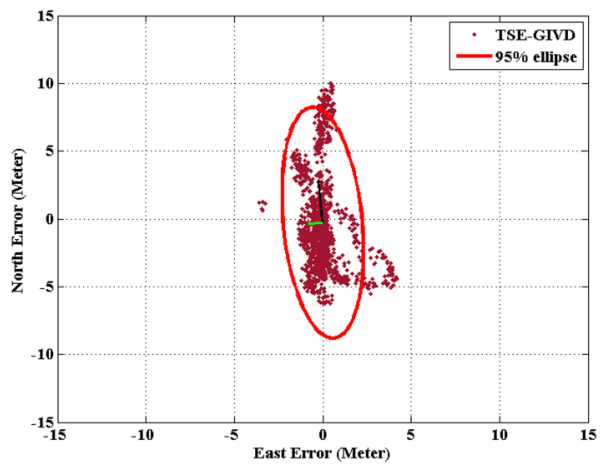

(c) TSE Correction (Reference GIVD Model)

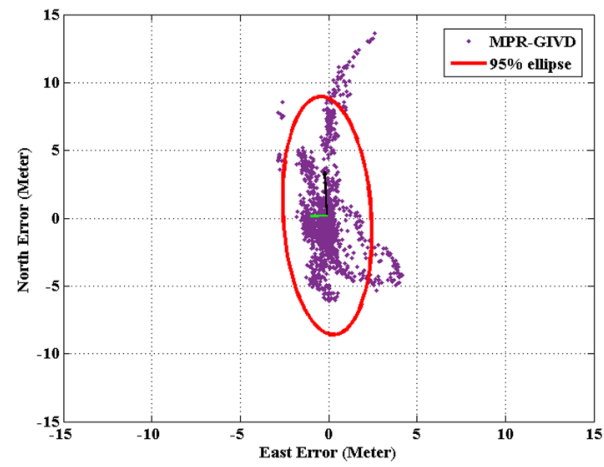

(e) MPR Correction (Reference GIVD Model)

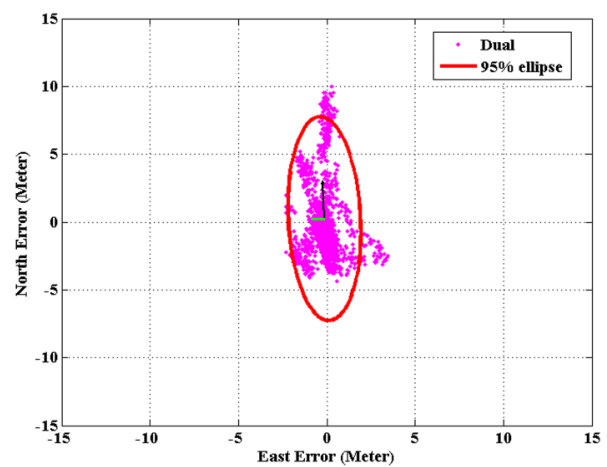

(g) Dual-frequency Correction

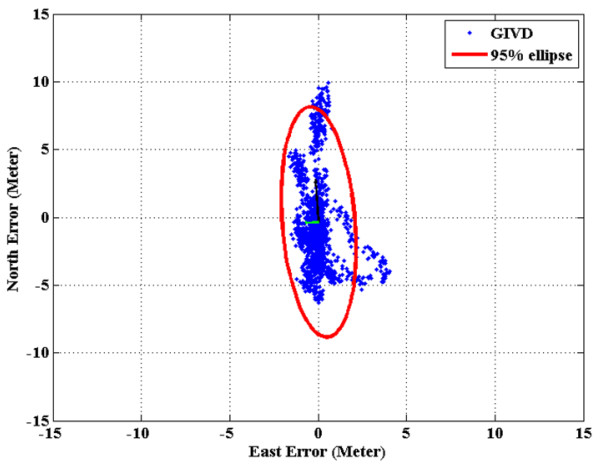

(b) GIVD Correction

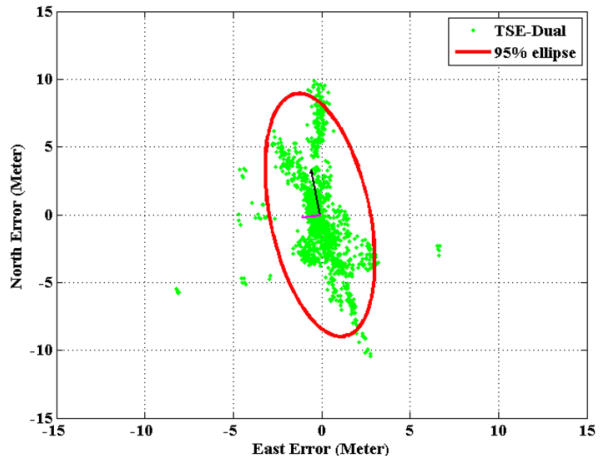

(d) TSE Correction (Reference Dual-Frequency)

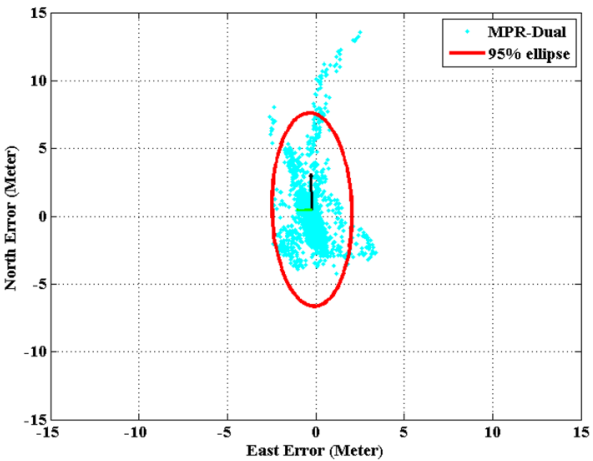

(f) MPR Correction (Reference Dual-Frequency)

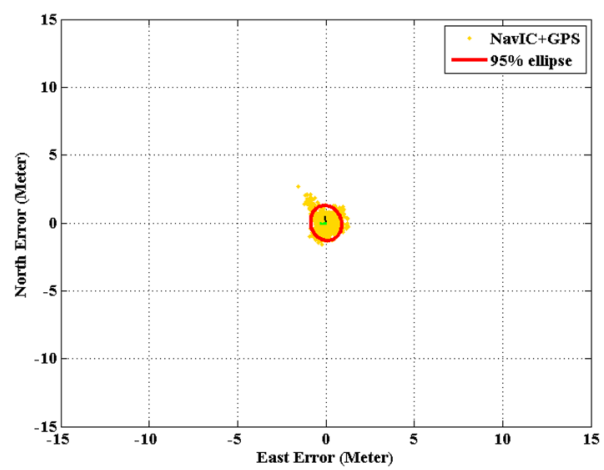

(h) NavIC L5-Band + GPS L1-Band 
4Fig. 9 NaviC East-North Error Correlation Plots for a Klobuchar Correction b GIVD Correction c TSE correction (Reference GIVD Model) dTSE Correction (Reference dual-frequency) e MPR correction (Reference GIVD Model) f MPR Correction (Reference dual-frequency) g dual-frequency correction $\mathbf{h}$ NavlC L5-Band+GPS L1-Band on $04 / 09 / 16\left(K_{p}=4+, A_{p}=27\right)$ at Rover Station-1

The correlation of east-north positioning errors for the above-discussed models (04/09/16, TOWC $=0 \mathrm{~s}-86,400 \mathrm{~s})$ is also evaluated in terms of $95 \%$ error ellipses, which are depicted in Fig. 9. We noted that compared to the singlefrequency Klobuchar corrections, the NavIC L5 band ENU errors are reduced after the dual-frequency corrections, i.e., the error ellipses are shorter compared to the Klobuchar corrections. The ENU error of the single-frequency GIVD model is almost the same as the ENU error of the dual-frequency correction, i.e., error ellipses are the same length. By comparison, we also conclude that for both cases ( $a$ and $b$ ), the ENU errors of NavIC L5 band rover station-1 using the local approaches (TSE and MPR) corrections are almost the same as the reference model on $04 / 09 / 16\left(K_{p}=4+, A_{p}=27\right)$, i.e., error ellipses are the same length. The shortest error ellipse is for the augmented NavIC and GPS system. The detailed positioning error comparison is listed in Table. 2.

We inferred from Table 2 that two sigma east-north errors are reduced to $\sim 2 \mathrm{~m}(2 \sigma \mathrm{E})$ and $\sim 3 \mathrm{~m}(2 \sigma \mathrm{N})$ after the dual-frequency corrections. The two sigma east-north errors for the GIVD model are similar to the dual-frequency method. The two sigma east-north errors of proposed local MPR approach are reduced $\sim 0.1-0.5 \mathrm{~m}$ compared to the TSE approach, and very similar to the reference model, only $0.1 \mathrm{~m}(2 \sigma \mathrm{E})$ difference is noted. The test was repeated at another single-frequency rover station (Station-2 refer Fig. 1. To validate the results, the analysis was carried out for a quiet day 04/01/18 $\left(K_{p}=1+, A_{p}=3\right.$, TOWC $=1 \mathrm{~s}-71,327 \mathrm{~s}$ ).

The estimated 3D positioning ENU errors using applied ionospheric corrections are compared in Table 3, and the MPR approach performance is noted similar to rover station-1,only $1-2 \mathrm{~m}(2 \sigma \mathrm{N})$ difference is indicated. Therefore, whether we considered the reference model as bias-free (i.e., multipath) single- frequency GIVD model or biased dual-frequency method, in both the Cases the proposed local MPR results are identical to their reference model. Hence, we inferred from the positioning errors results of two rover stations (Case-II) that if the local approach coefficients are generated by the bias-free reference dual-frequency method then also the ionospheric delays estimated by the proposed local MPR approaches at single-frequency rover stations is as accurate as reference dual-frequency model, and reducing the hardware cost of extra frequency.

\subsection{Changing rover location over a local region}

To determine the working range of the Local model, the rover location is varied, as shown in Fig. 10. In Fig. 10, the rover station is marked in green color and indicated by letter $\mathrm{T}$, where the reference is marked with pink color. The latitude and longitude of the reference and the different rover station locations are listed in Table 4.

The distance between rover station $\mathrm{T} 1$ and the reference station is $0.38 \mathrm{~km}$. The rover T2-T5 is located on the South-West side of the reference position. Similarly, the T6-T12 is located on the North-East side of the reference station. The distance from the reference station to rover $\mathrm{T} 2$ is $3.41 \mathrm{~km}$, to rover T3 is $6.48 \mathrm{~km}$, to rover T4 is $9.07 \mathrm{~km}$, to rover T5 is $11.1 \mathrm{~km}$. Similarly, distance from a reference station to rover $\mathrm{T6}$, rover $\mathrm{T}$, rover $\mathrm{T} 8$, rover $\mathrm{T}$, rover $\mathrm{T} 10$, rover $\mathrm{T} 11$, and rover $T 12$ are $1.16 \mathrm{~km}, 2.65 \mathrm{~km}, 3.75 \mathrm{~km}, 5.73 \mathrm{~km}$, $7.1 \mathrm{~km}, 7.18 \mathrm{~km}$, and $8.09 \mathrm{~km}$, respectively.

The difference between the reference dual-frequency model and the proposed approach is calculated with coefficient broadcast intervals of $1 \mathrm{~min}$ and $5 \mathrm{~min}$ (post-processing mode). Figure 11 shows the proposed approaches (i.e., local TSE and MPR) performance at the nearest distance $(T 1=0.38 \mathrm{~km}$ and $T 6=1.16 \mathrm{~km})$, middistance $(T 3=6.48 \mathrm{~km}$ and $T 9=7.1 \mathrm{~km})$, and extreme distance $(T 5=11.1 \mathrm{~km}$ and $T 12=8.09 \mathrm{~km})$. We observed that the $\mathrm{T} 1$ rover station is located very near the reference receiver. Hence, the estimation of the proposed TSE and MPR approaches with coefficients broadcast $5 \mathrm{~min}$ and $1 \mathrm{~min}$ are similar.

Also, as the distance between reference and rover station increases, performance errors are increased. We inferred from the error performance of rover locations that the proposed MPR and TSE approach performed well, with a significant error of up to $0.4 \mathrm{~m}$, which is further reduced to $0.2 \mathrm{~m}$ or more by reducing the coefficients broadcast interval of five minutes to one minute. Therefore from the result analysis, we noted that single frequency MPR approaches effectively corrected the ionospheric correction and reduced the positioning errors up to 99\% $(0.1 \mathrm{~m}$ East error) and 90\% (1 $\mathrm{m}$ North error) similar to the reference model.

\section{Conclusions}

To estimate the precise ionospheric delay within a local region $(10 \mathrm{~km})$ using a single-frequency $\mathrm{NavlC}$ receiver, a local MPR approach is proposed in this paper. For the validation of the proposed approach, two Cases considered (I) single static NavIC receiver and (II) two NavIC receivers (One considered as a reference and another as a rover) separated by $3.1 \mathrm{~km}$. The performance of 
Table 2 Statistical Positioning Error Analysis at Rover Station-1
Table 3 Statistical Positioning Error Analysis at Rover Station-2

\begin{tabular}{lll}
\hline Rover Station-1 $\left(21^{\circ} 8^{\prime} 23.5993^{\prime \prime} \mathrm{N}, 72^{\circ} 47^{\prime} 39.6024^{\prime \prime} \mathrm{E}\right)$ & & \\
\hline TOWC $=0-86,400 \mathrm{~s}, 04 / 09 / 16\left(\mathrm{~K}_{\mathrm{p}}=4+, \mathrm{A}_{\mathrm{p}}=27\right)$ & & North-Error \\
\hline Methods/ Algorithm & East-Error 20E(meter) & $2 \sigma \mathrm{N}$ (meter) \\
\hline NavIC Klobuchar & 3.56 & 9.14 \\
NavIC GIVD & 1.74 & 6.94 \\
NavIC TSE (GIVD Reference Model) & 1.91 & 6.96 \\
NavIC TSE (Dual-Frequency Reference Model) & 2.54 & 7.34 \\
NavIC MPR (GIVD Reference Model) & 1.87 & 6.85 \\
NavIC MPR (Dual-Frequency Reference Model) & 2.08 & 7.21 \\
NavIC Dual-Frequency & 1.71 & 6.14 \\
NavIC Dual-Frequency+GPS & 0.74 & 1.06
\end{tabular}

\begin{tabular}{|c|c|c|}
\hline \multicolumn{3}{|c|}{ Rover Station-2 (21ㅇ' $\left.9^{\prime} 20.7590^{\prime \prime} \mathrm{N}, 72^{\circ} 49^{\prime} 20.1275^{\prime \prime} \mathrm{E}\right)$} \\
\hline \multicolumn{3}{|c|}{ TOWC $=369,158 \mathrm{~s}-431,941 \mathrm{~s}, 04 / 01 / 18\left(K_{p}=1+, A_{p}=3\right)$} \\
\hline Methods/ Algorithm & East-Error 2бE(meter) & $\begin{array}{l}\text { North-Error } \\
2 \sigma N \text { (meter }\end{array}$ \\
\hline NavIC GIVD & 1.82 & 3.34 \\
\hline NavIC TSE (GIVD Reference Model) & 1.73 & 4.93 \\
\hline NavIC TSE (Dual-Frequency Reference Model) & 2.22 & 5.77 \\
\hline NavIC MPR (GIVD Reference Model) & 1.62 & 5.15 \\
\hline NavIC MPR (Dual-Frequency Reference Model) & 2.18 & 4.48 \\
\hline NavIC Dual-Frequency & 2.12 & 3.42 \\
\hline
\end{tabular}

the local MPR method was verified under the influence of the geomagnetic storm on September 8, 2017. The results of the single-frequency global model (Klobuchar), the regional model (GIVD), and the local proposed TSE and MPR approaches were analyzed and compared. By comparing the residual errors of all applied models in all observed stations, we found that the proposed singlefrequency local MPR approach similar to the reference dual-frequency method in the Case of an intense geomagnetic storm.

In Case II, to estimate the ionospheric delay accurately, the rover NavlC receiver (single-frequency) located in $<10 \mathrm{~km}$ area has received the coefficient transmitted by the reference NavIC receiver (dual-frequency) of SVNIT Surat station. From the comparative analysis of the NavIC
L5-band ionospheric delay and positioning errors (ENU) ellipses, it is observed that the proposed MPR approach performs (symmetrical coefficients) worked better (i.e., remove spikes that occur in TSE) compare to TSE (asymmetrical coefficients). It is also observed that a single-frequency MPR approach is close to 99\% (0.1 m East error) and $90 \%$ (1 $\mathrm{m}$ North error) similar to the reference model, which can effectively correct the ionospheric correction and reduce the positioning error.

The MPR and TSE approaches are also examined at a different geographical location in the local $10 \mathrm{~km}$ region. It has been observed from NavlC position estimation that the MPR approach is related to the performance of the reference dual-frequency receiver, and only a residual error of $\sim 0.2-0.4 \mathrm{~m}$ is observed, while 
Fig. 10 The Locations of the local Rover NavIC Stations for the Performance Observations of Proposed TSE and MPR Approach

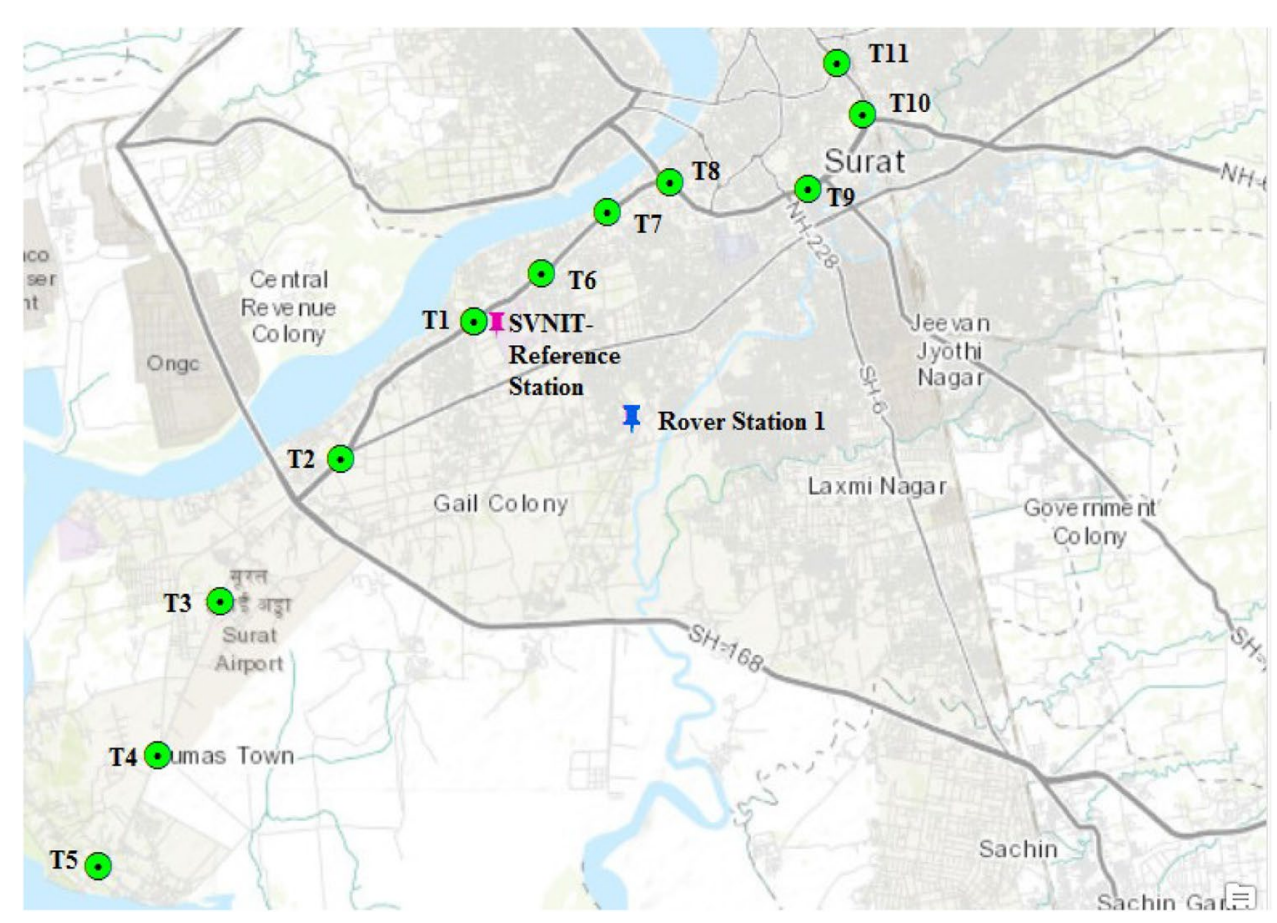

Table 4 The Position of NavIC Reference and Rover Station (T1-T12)

\begin{tabular}{lll}
\hline Station Name & Latitude & Longitude \\
\hline Reference Receiver & $21^{\circ} 9^{\prime} 50.0926^{\prime \prime} \mathrm{N}$ & $72^{\circ} 47^{\prime} 1.2511^{\prime \prime} \mathrm{E}$ \\
Rover Station Location-T1 & $21^{\circ} 9^{\prime} 52.9641^{\prime \prime} \mathrm{N}$ & $72^{\circ} 46^{\prime} 48.5853^{\prime \prime} \mathrm{E}$ \\
Rover Station Location -T2 & $21^{\circ} 8^{\prime} 39.7767^{\prime \prime} \mathrm{N}$ & $72^{\circ} 45^{\prime} 29.9442^{\prime \prime} \mathrm{E}$ \\
Rover Station Location -T3 & $21^{\circ} 6^{\prime} 2.5119^{\prime \prime} \mathrm{N}$ & $72^{\circ} 43^{\prime} 42.2377^{\prime \prime} \mathrm{E}$ \\
Rover Station Location -T4 & $21^{\circ} 7^{\prime} 24.7309^{\prime \prime} \mathrm{N}$ & $72^{\circ} 47^{\prime} 19.1988^{\prime \prime} \mathrm{E}$ \\
Rover Station Location -T5 & $21^{\circ} 5^{\prime} 9.9261^{\prime \prime} \mathrm{N}$ & $72^{\circ} 43^{\prime} 7.6994^{\prime \prime} \mathrm{E}$ \\
Rover Station Location -T6 & $21^{\circ} 10^{\prime} 18.6099^{\prime \prime} \mathrm{N}$ & $72^{\circ} 47^{\prime} 27.4439^{\prime \prime} \mathrm{E}$ \\
Rover Station Location -T7 & $21^{\circ} 10^{\prime} 59.4475^{\prime \prime} \mathrm{N}$ & $72^{\circ} 48^{\prime} 5.9675^{\prime \prime} \mathrm{E}$ \\
Rover Station Location -T8 & $21^{\circ} 11^{\prime} 6.6919^{\prime \prime} \mathrm{N}$ & $72^{\circ} 48^{\prime} 42.3747^{\prime \prime} \mathrm{E}$ \\
Rover Station Location -T9 & $21^{\circ} 11^{\prime} 3.2669^{\prime \prime} \mathrm{N}$ & $72^{\circ} 50^{\prime} 4.1708^{\prime \prime} \mathrm{E}$ \\
Rover Station Location -T10 & $21^{\circ} 11^{\prime} 42.1814^{\prime \prime} \mathrm{N}$ & $72^{\circ} 50^{\prime} 36.9762^{\prime \prime} \mathrm{E}$ \\
Rover Station Location -T11 & $21^{\circ} 12^{\prime} 47.1159^{\prime \prime} \mathrm{N}$ & $72^{\circ} 50^{\prime} 20.1822^{\prime \prime} \mathrm{E}$ \\
Rover Station Location -T12 & $21^{\circ} 13^{\prime} 46.2268^{\prime \prime} \mathrm{N}$ & $72^{\circ} 50^{\prime} 9.7950^{\prime \prime} \mathrm{E}$ \\
\hline
\end{tabular}

the MPR method eliminates the spikes that occur in the TSE approach. Therefore, the ionospheric correction applied by the local MPR approach not only improves the performance of the rover NavIC receivers in the local region but also reduce the computational cost and additional frequency if errors of up to $0.4 \mathrm{~m}$ are tolerated. In the future, if the dual-frequency NavIC receiver is installed with the Base Trans-receiver Station (BTS) of the mobile communication network, then, using this approach, mobile users (having NavIC-L5 band facility) can receive the local MPR approache coefficients from BTS (having dual-frequency NavIC receiver facility) to estimate the ionospheric correction for best mobile positioning in the local region. 


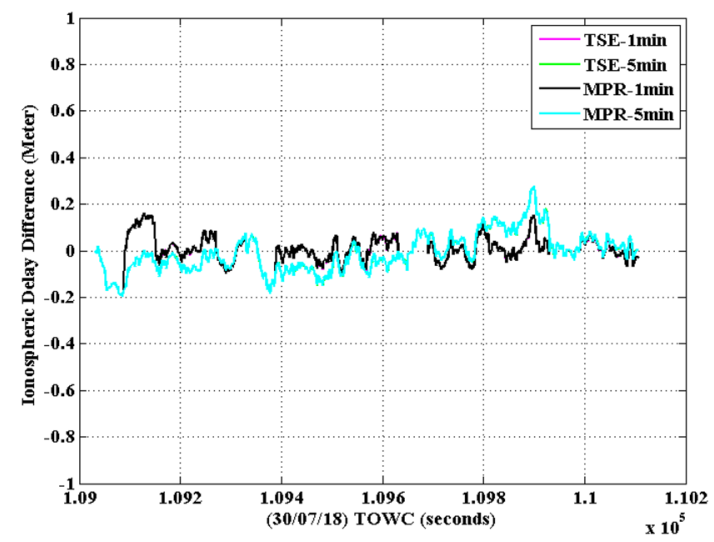

(a) $\mathrm{T} 1(0.38 \mathrm{~km}$ away from reference $)$

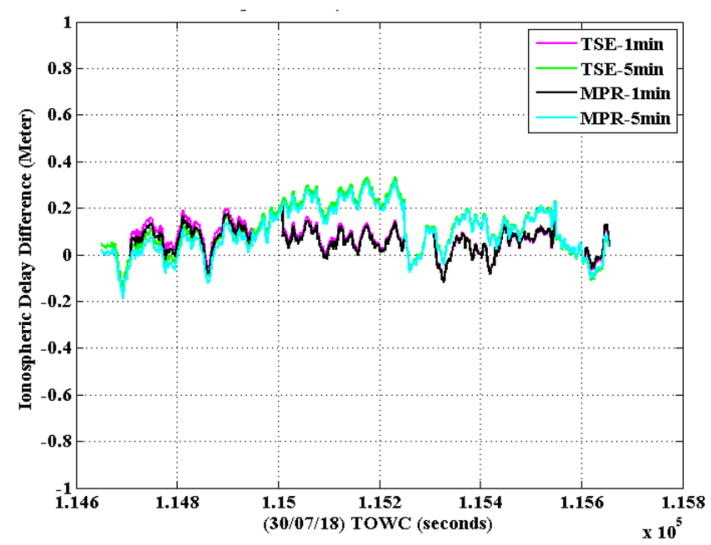

(c) $\mathrm{T} 3(6.48 \mathrm{~km}$ away from reference)

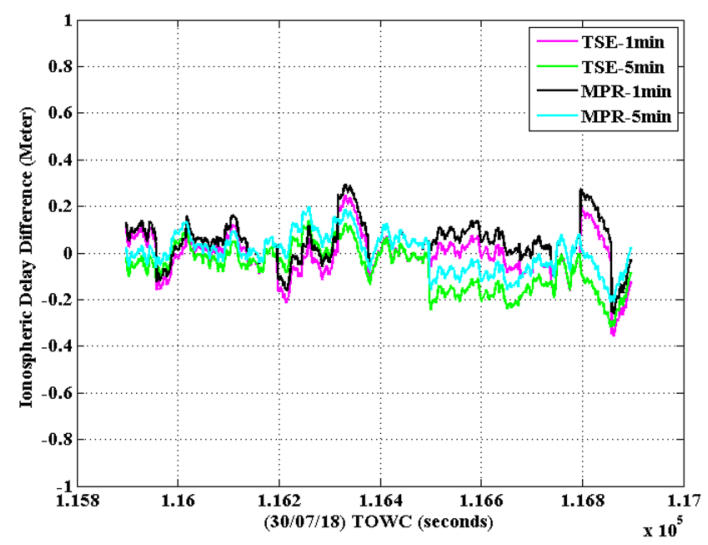

(e) $\mathrm{T} 5$ (11.1 km away from reference)

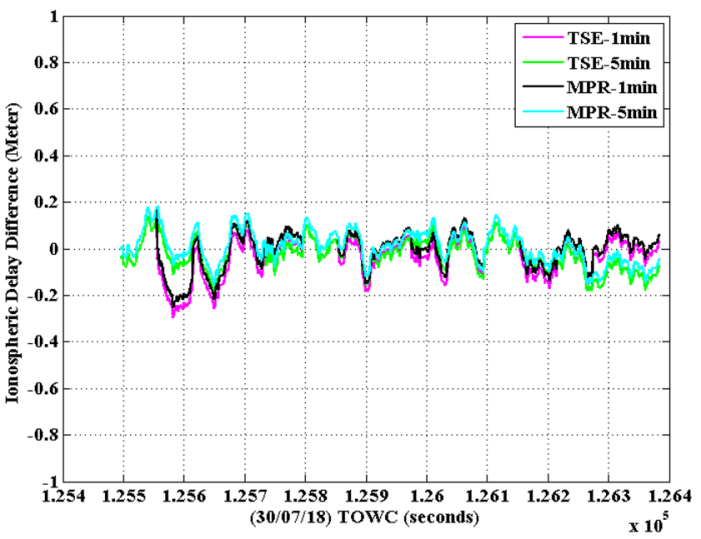

(b) T6 (1.16 km away from reference)

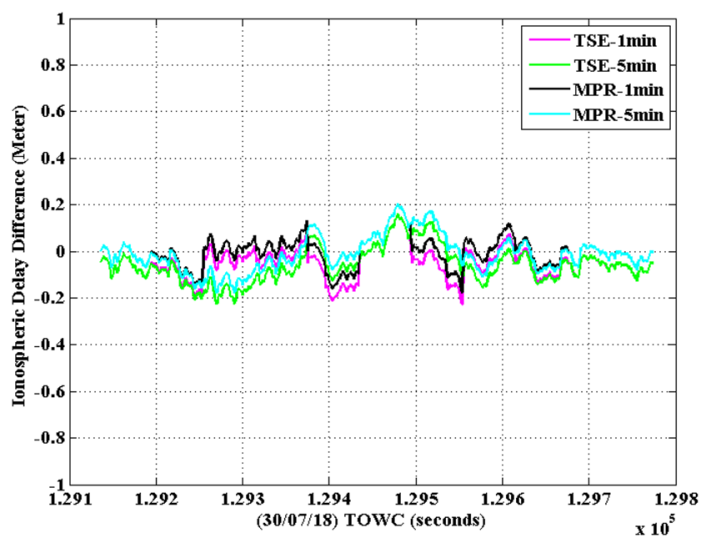

(d) $\mathrm{T} 9(5.73 \mathrm{~km}$ away from reference $)$

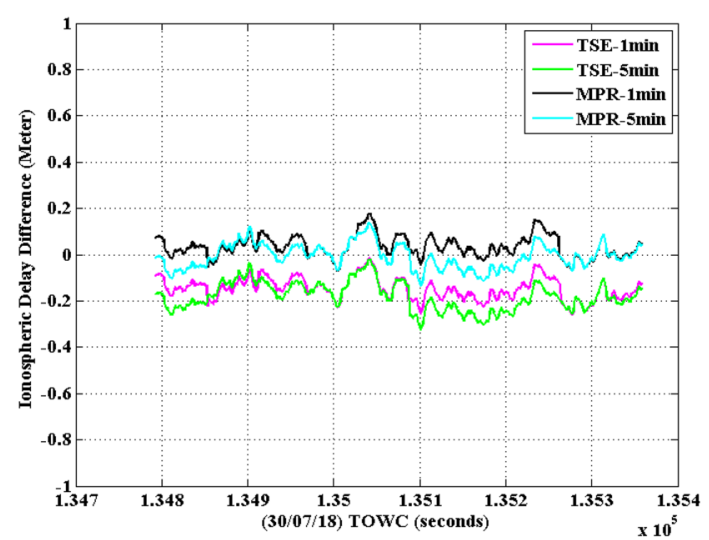

(f) T12 (8.09 km away from reference)

Fig. 11 Performance Observations of Proposed TSE and MPR Approach at the nearest distance $\mathbf{a} \mathrm{T} 1=0.38 \mathrm{~km}) \mathbf{b}$ T6=1.16 km, mid-distance $\mathbf{c}$ $\mathrm{T} 3=6.48 \mathrm{~km} \mathbf{d} \mathrm{T} 9=7.1 \mathrm{~km}$, and extreme distance $\mathbf{e} 5=11.1 \mathrm{~km} \mathbf{f} \mathrm{T} 12=8.09 \mathrm{~km}$

Acknowledgments This research work is part of the Announcement of Opportunity (AO) project (NGP-8) sponsored by SAC, ISRO, Ahmedabad, Gujarat, India.

\section{Compliance with ethical standards}

Conflicts of interest On behalf of all authors, I state that there is no conflict of interest. 


\section{References}

1. Desai MV, Jagiwala D Shah SN (2016a) Impact of dilution of precision for position computation in Indian regional navigation satellite system. In: International conference on advances in computing, communications and informatics (ICACCI), pp 980-986. https://doi.org/10.1109/ICACCI.2016.7732172

2. Desai MV, Shah SN (2017) Analysis of ionospheric correction approach for IRNSS/NavIC system based on loT platform. Int Conf Future Internet Technol Trends. 174-183. https://doi. org/10.1007/978-3-319-73712-6_18

3. Desai MV, Shah SN (2019b) An observational review on influence of intense geomagnetic storm on positioning accuracy of NavIC/IRNSS system. IETE Tech Rev 37(3):1-15. https://doi. org/10.1080/02564602.2019.1599739

4. Desai MV, Shah SN (2020) Case study: performance observation of NavIC ionodelay and positioning accuracy. IETE Tech Rev 1-11. https://doi.org/10.1080/02564602.2020.1723445

5. Desai MV, Shah SN (2019c) Estimation of ionospheric delay of NavIC/IRNSS signals using the Taylor series expansion. J Space Weather Space Clim 9:1-17. https://doi.org/10.1051/swsc/20190 23

6. Desai MV, Shah SN (2018e) Impacts of intense geomagnetic storms on NavIC/IRNSS system. Ann Geophys 61(5):1-15. https ://doi.org/10.4401/ag-7856

7. Desai MV, Shah SN (2018d) The GIVE ionospheric delay correction approach to improve positioning accuracy of NavIC/IRNSS single frequency receiver. Curr Sci 114(8):1665-1676. https:// doi.org/10.18520/cs/v114/i08/1665-1676

8. Fujita S, Kubo Y, Sugimoto S (2009b) lonosphere total electron content estimation based on GNSS regression models at known positions. Int J Innov Comput Inf Control 5(1):139-152

9. Fujita S, Yamamoto H, lura T, Kubo Y, Sugimoto S (2010) Modeling ionosphere VTEC over Japan based on GNSS regression models and GEONET. Int J Innov Comput Inf Control 6(1):155-169

10. Hopfield HS (1971) Tropospheric effect on electromagnetically measured range: Prediction from surface weather dat. Radio sci 6(3):357-367

11. He Y, Bilgic $A(2011$ b) Iterative least squares method for global positioning system. Adv Radio Sci 9:203-207

12. ICD. (2018a) "IRNSS signal in space, ISRO-ISAC, V 1.1.", https:// www.isro.gov.in/irnss-programme, last accessed 25 August 2018.

13. ISRO.(2018b), "Indian space research organization (ISRO).",https://www.isro.gov.in/applications/satellite-navigation- programme, last accessed 25 August 2018.
14. Jagiwala DD, Shah SN (2018c) Perception and reduction of Wi-Fi interference on NavIC signals. IET Radar Sonar Navig 114(11): 2273-2280. https://doi.org/10.1049/iet-rsn.2018

15. Kumar PN, Sarma AD, Reddy AS (2014) Modelling of ionospheric time delay of global positioning system (GPS) signals using taylor series expansion for GPS aided geo augmented navigation applications. IET Radar Sonar Navig 8(9):1081-1090

16. Lineswala PL, Shah SN (2019a) Jamming: The probable menace to NavIC. IET Radar Sonar Navig 13(6):1039-1044. https://doi. org/10.1049/iet-rsn.2018.5429

17. Ratnam DV, Dabbakuti JRKK, Lakshmi NVVNJS (2018f) Improvement of Indian-regional klobuchar ionospheric model parameters for single-frequency GNSS users. IEEE Geosci Remote Sens Lett 15(7):971-975

18. Ratnam DV, Sarma AD (2006a) Modeling of Indian ionosphere using MMSE estimator for GAGAN applications. J Ind Geophys Uni 10(4):303-312

19. Ratnam DV, Sarma AD (2012) Modeling of low-latitude ionosphere using GPS data with SHF model. IEEE Trans Geosci Remote Sens 50(3):972-980

20. Ratnam DV, Sarma AD, Srinivas VS, Sreelatha P (2011a) Performance evaluation of selected ionospheric delay models during geomagnetic storm conditions in low-latitude region. Radio Sci 46(3):307-315

21. Sarma AD, Prasad N, Madhu T (2006b) Investigation of suitability of grid-based ionospheric models for GAGAN. IET Electron Lett 42(8):478-479

22. Sarma AD, Ratnam DV, Reddy DK (2009a) Modelling of low-latitude ionosphere using modified planar fit method for GAGAN. IET Radar Sonar Navig 3(6):609-619

23. Shukla AK, Das S, Shukla AP, Palsule VS (2013a) Approach for near-real-time prediction of ionospheric delay using klobuchar-like coefficients for indian region. IET Radar Sonar Navig $7(1): 67-74$

24. Sinha $P(2013 b)$ Multivariate polynomial regression in data mining: methodology, problems and solutions. Int J Sci Eng Res 4(12):962-965

25. Srinivas VS, Sarma AD, Achanta HK (2016b) Modeling of ionospheric time delay using anisotropic idwwith jackknife technique. IEEE Trans Geosci Remote Sens 54(1):513-519

Publisher's Note Springer Nature remains neutral with regard to jurisdictional claims in published maps and institutional affiliations. 